

\title{
National Seismic Stations Transducers and Filters
}

\author{
P. W. Rodgers
}

M. Hummull

Manuscript date: January 13. 1981

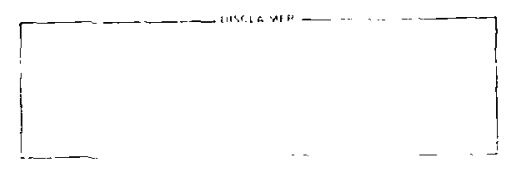

I AWRENCE LIVERMORE LABORATOR Y Liniversity of California • Livermore. California • 94550 


\section{CONTENTS}

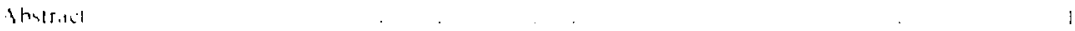

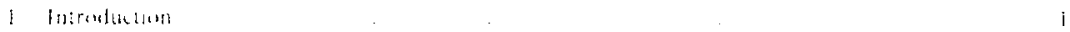

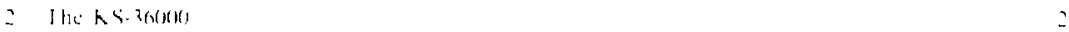

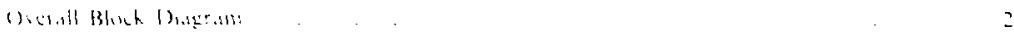

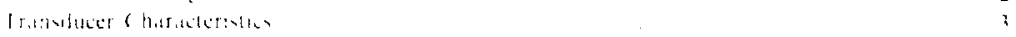

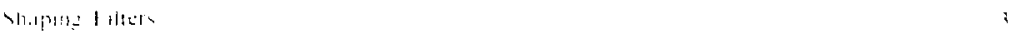

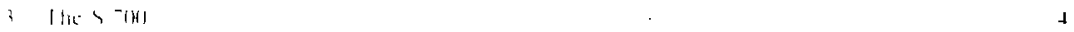

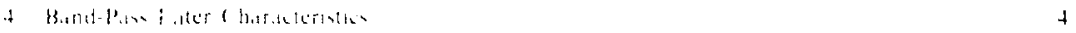

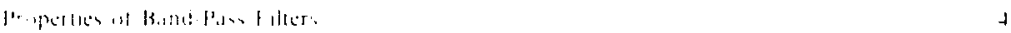

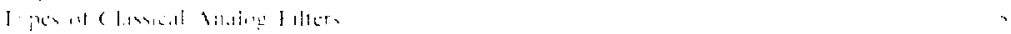

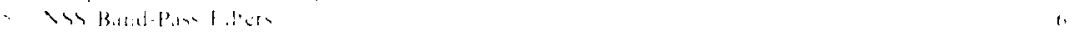

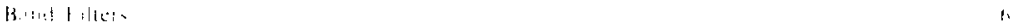

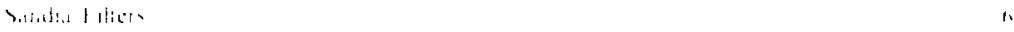

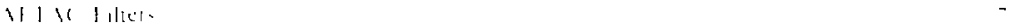

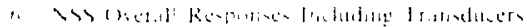

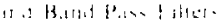

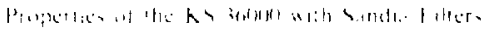

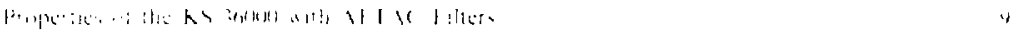

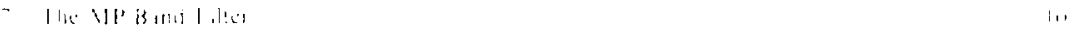

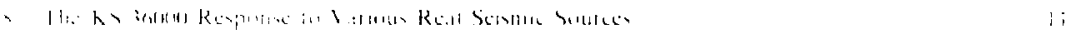

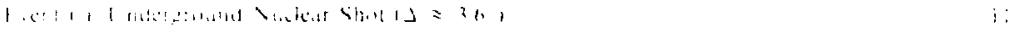

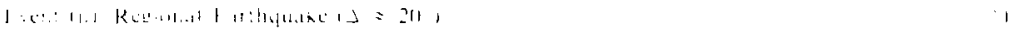

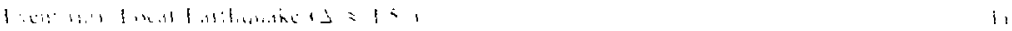

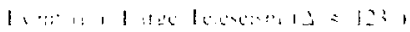

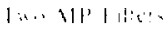

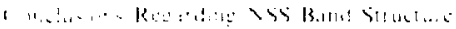

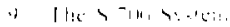

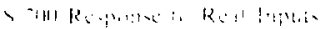

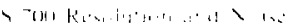

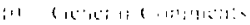

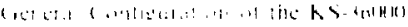

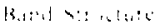

like $>$ - $i$ itt

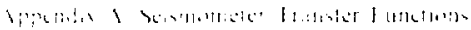

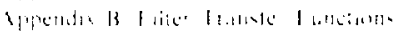

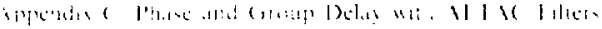

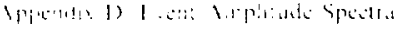




\section{National Seismic Stations \\ Transducers and Filters}

\section{ABSTRAC}

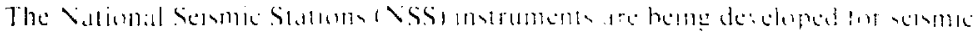

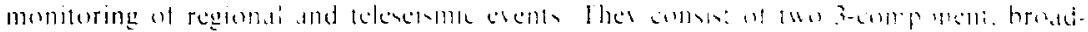

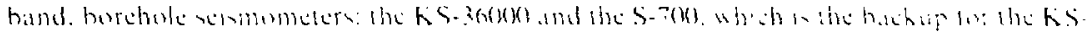

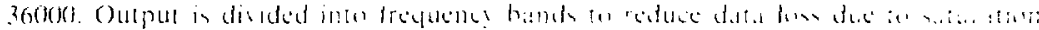

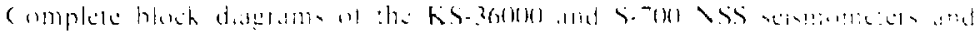

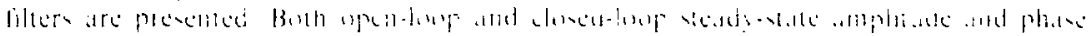

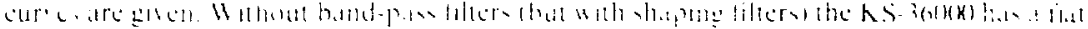

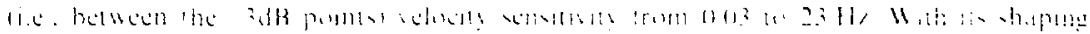

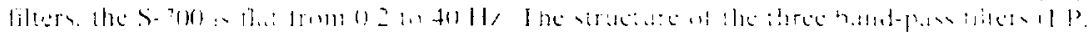

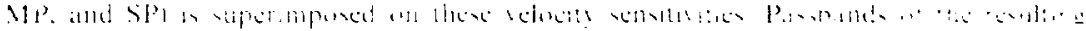

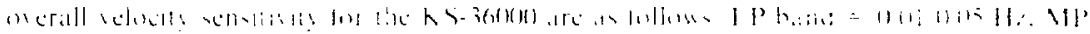

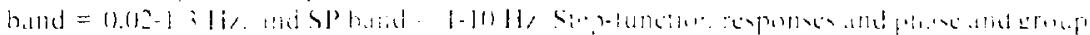

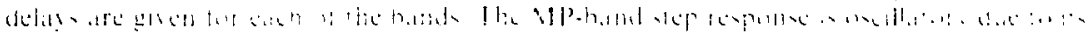

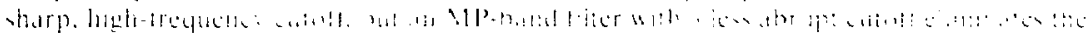
ancillutian.

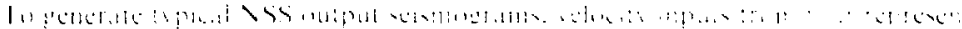
l.1160 -

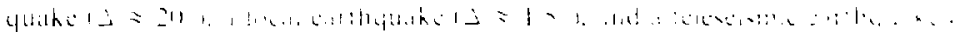
lhe a 10)

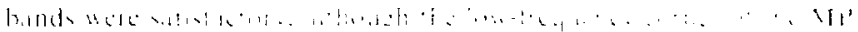

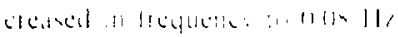

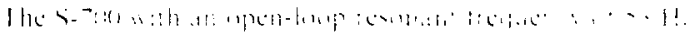

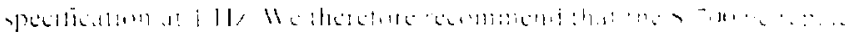

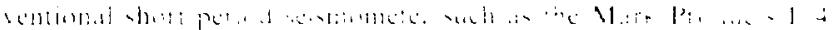

\section{(HIPIFR I: MIROH) (IIO)}

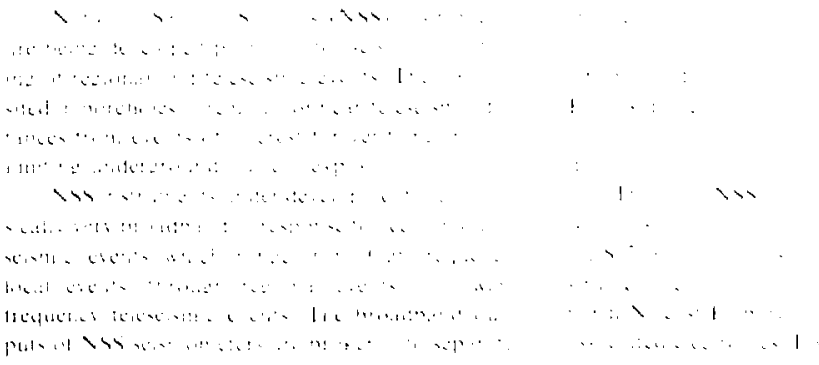




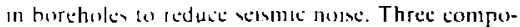
ments ate und beciase the ereatly increitse the abilic: w idenerly partscular wate typen.

the repors present the steirds-state vinutondal

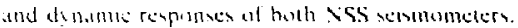

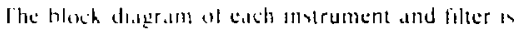

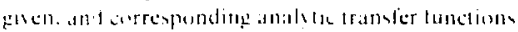
itrs colntand an lne tppendice

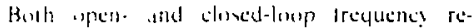

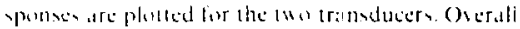

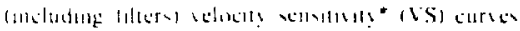

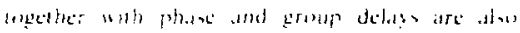
prencricis!

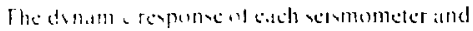

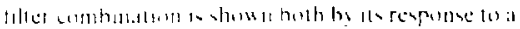

step function in velocits and its responne to seismic inputs Representative sesmle inputs are velocitygrams of locial. regonnill. and telesejsmic erents as recorded by the 1.I. Nl dignal seisme network.

Dats for thex eomputitions were taken from the sugue? ! 1. 1979 and tehruary 27. 1980 informal report, uf H. B Durham isandas): "VSSModel Il. Stami Data Sintem Responxe funkImns." Tratrater funclom lor the S-700 were laken Irom the laller report and thome for the KS-36000 from the furmer: therture the revponace fur the $k S$ -

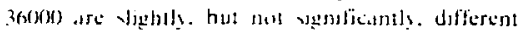
Irwen the curren sersion

\section{CHAPTER 2: THE KS-36000}

\section{ONFRAII. BIOC'K I)IAGRAM}

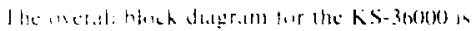

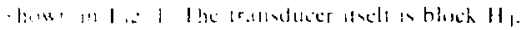

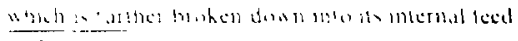

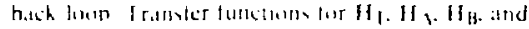

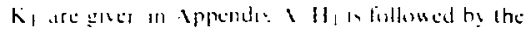

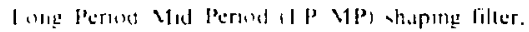

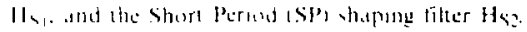

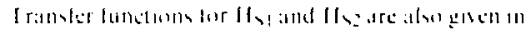

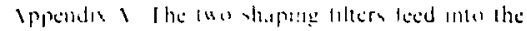
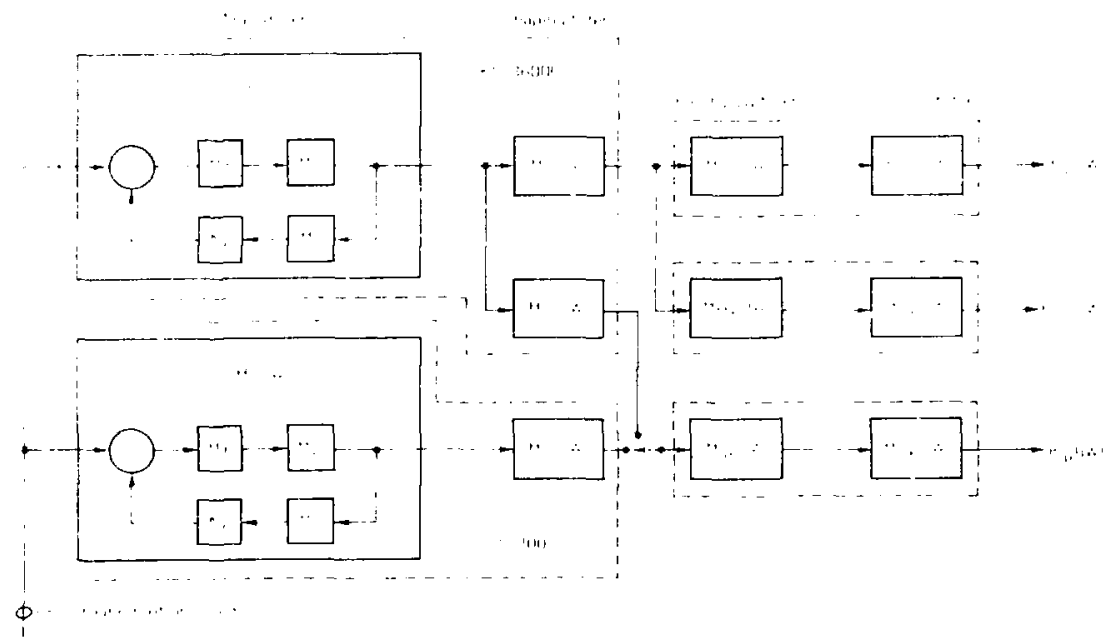

Fl(i. 1. () verall block diagram for U.S scismographs showing feedback loops for the transducers. ,haping filters, band-pass filters, and common filters. 
three major band-pass filters $H_{L P}, H_{M P}$. and $H_{S P}$ which. in turn. are followed by the three common filters. $H_{C l}$. Transfer functions for the band-pass and comiron filters are listed in Appendix B.

\section{TRANSDUCER CHARACTERISTICS}

The open-loop freyuency-renponse curve. $\mathrm{H}_{\mathrm{T}} \mathrm{H}_{\mathrm{A}} \mathrm{H}_{v \mathrm{~K}}$ is given in Fig. 2. The peat at approsimately $0.2 \mathrm{~Hz}$ is due to the resonance of the lightus damped is $=0.014171$ spring-mans stitem of the seisnometer iscli. The closed-forp accelerdion response uhich is llat wut $1023 \mathrm{H} /(-3 \mathrm{~dB})$ with al

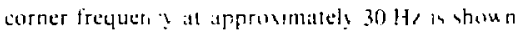
in lig. 3 .

\section{SHAPING; FIITERS}

Figures 4 and 5 shen the frequency reaporse of the tuo shaping filters. Hs and $H_{5}$. The purpese of $H_{s 1}$ is lo calse the vScurre of $H_{1}$ in liall off as $-f^{3}$ at frequencies below $0.01 \mathrm{H}$. Without $\mathrm{H}_{\mathrm{Sl}}$. il uould fall ofl as fi. The resulang onerdll VS curse for the transduece (sit, Hsil is shoun in Fig. 6. It rises whth it +3 slope (corresponding $t: 3 f^{3}$ ) belew its low-frequency corner all $0.0105 \mathrm{H} / \mathrm{l}=1.95 \mathrm{secj}$. 11

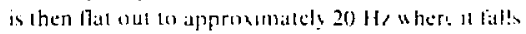
olf with at -4 tope. The frequency response of LP and MP hands uill he superimposed on this curve to ohtain the overall V'S curses lor the KS-36000.

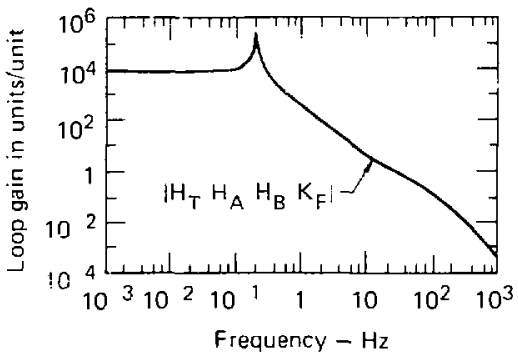

FIG. 2. KS-360)0 open-loop frequency-responsc curve peaks at approximarely $0.2 \mathrm{~Hz}$ due to mechanical resonance of the pendulum.

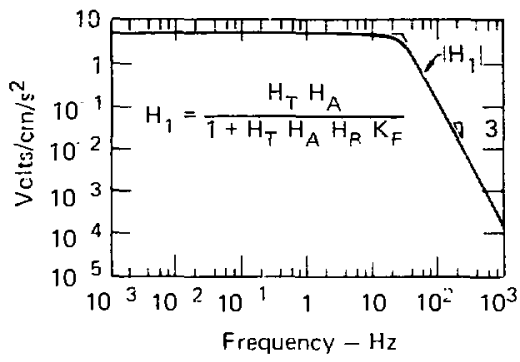

FIG. 3. K $\$-36000$ closed loop acceleration response is flat to $23 \mathrm{ilz}$.

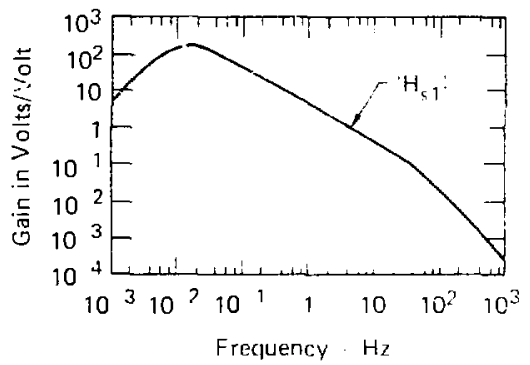

FI(:. f. Shaping filter, $H_{-1}$, frequency response.

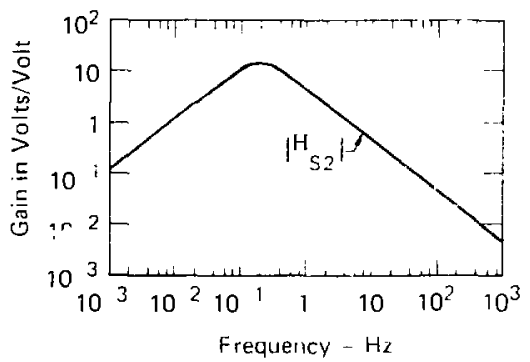

FIC, 5, Shaping filter, $\mathrm{H}_{\mathrm{S2}}$, frequency responses. 


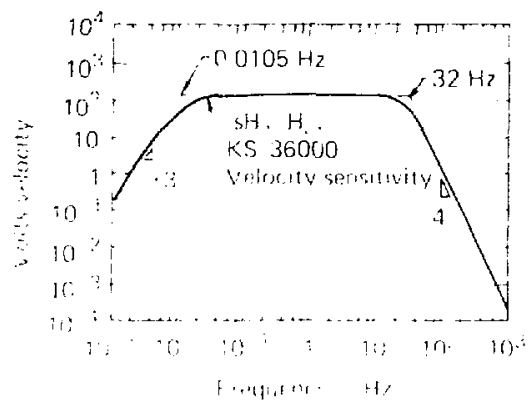

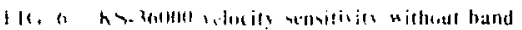
filare for \& I' and VI, band.

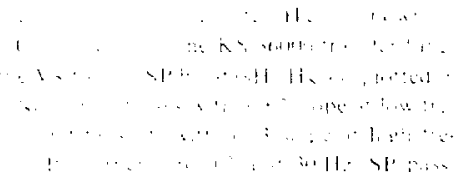

\section{CHAPTER 3: IHF S-70II}

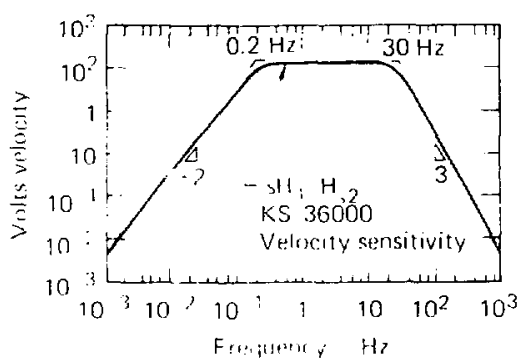

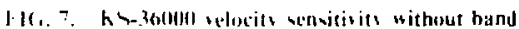
Fileors far si' band.

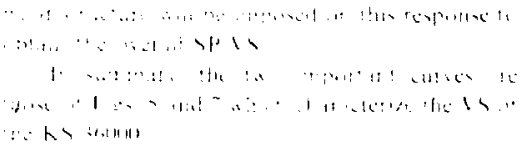

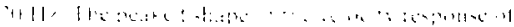

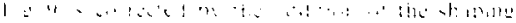

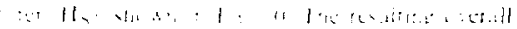

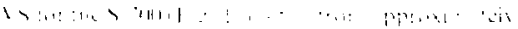

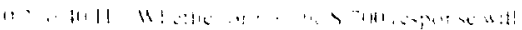

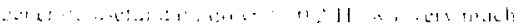

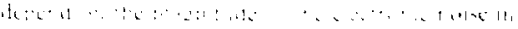

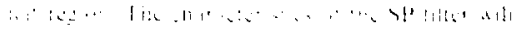

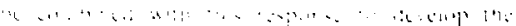

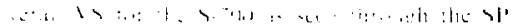

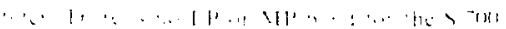

\section{(HAPTR 4: BAND-PISS FIITHR CHARACIHRISTICS}

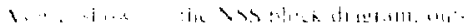

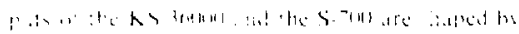

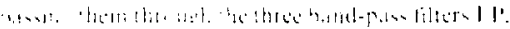

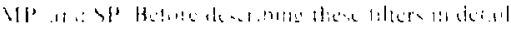

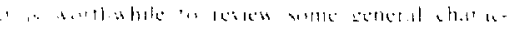

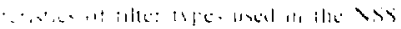

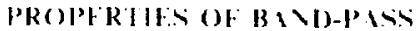
HII $1 \mathrm{FR}$

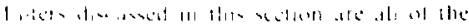
14: 


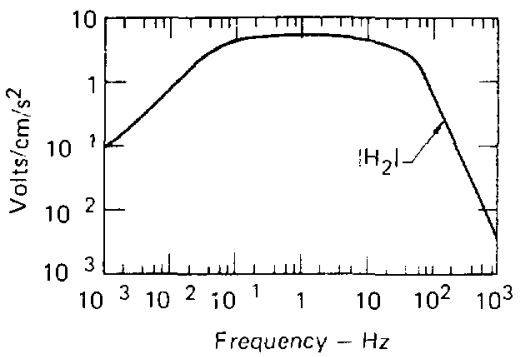

FIC. 8. S-7lill closed-loop acceleration response.

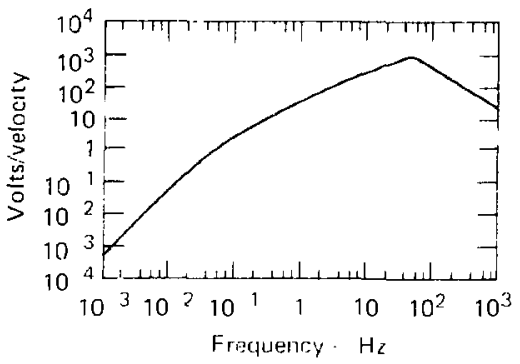

FIC;. 9. S-7601 closed-loop relocity response.

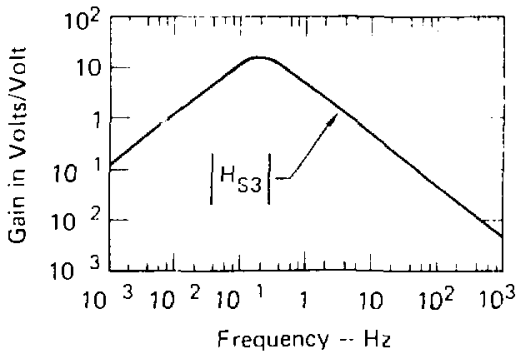

FIC. 10. Shaping filter, $\mathrm{H}_{S, 3}$ for $\mathrm{S}-7011$.

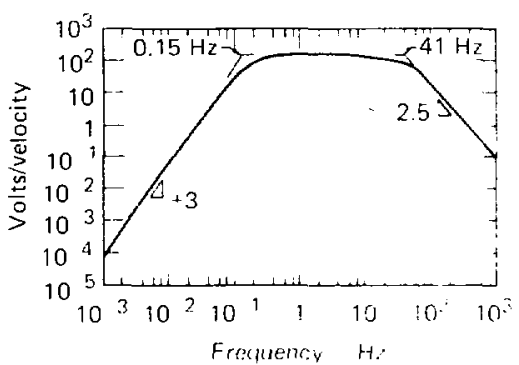

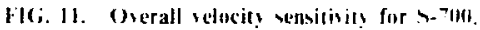

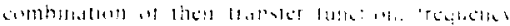

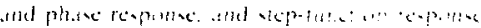

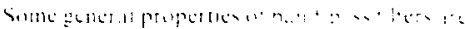
"wikn.

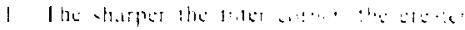

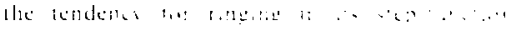
ricyinge

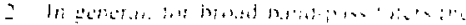

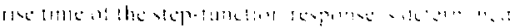

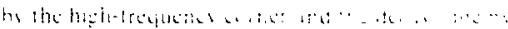

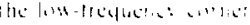

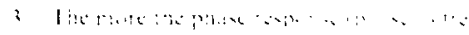

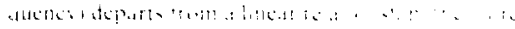

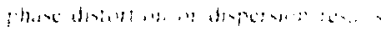

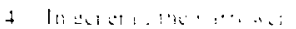

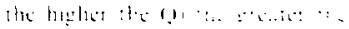

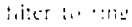

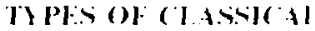

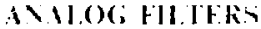

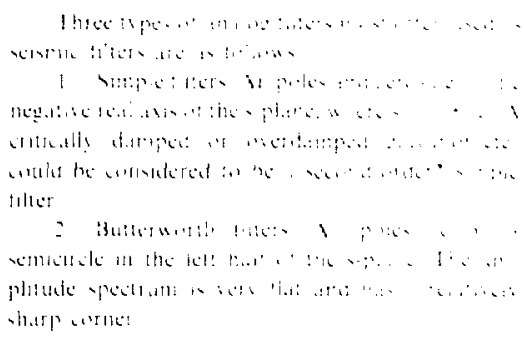

$-1 \cdots, \ldots, 16 ;$

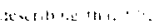


I BI.F. 1. Summars of simple, Butterworth. and B,encl filter characteristics.

\begin{tabular}{|c|c|c|c|}
\hline & simplu filter & Butsermath fills & Rament filted \\
\hline $\begin{array}{l}\text { vhape of apeciral } \\
\text { amplitude response }\end{array}$ & 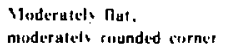 & $\begin{array}{l}\text { ters flat. } \\
\text { vherp eurner }\end{array}$ & $\begin{array}{l}\text { ler vluping. } \\
\text { ser reunded cormer }\end{array}$ \\
\hline $\begin{array}{l}\text { 1) berahaest in } \\
\text { wep echpernet }\end{array}$ & $\operatorname{Van}$ & Sloderale & It. lomit \\
\hline Ithas risturtitn" & Maderalu. & Muint a blt & l Iatul \\
\hline
\end{tabular}

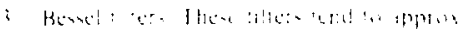

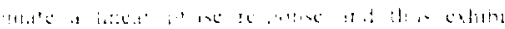

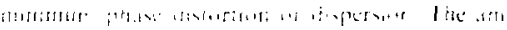

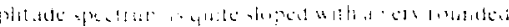
W $1 \mathrm{i} \cdot * 1$

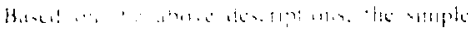
...

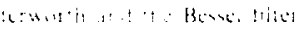

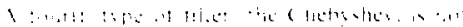

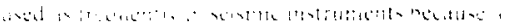

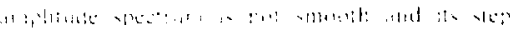

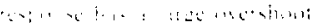

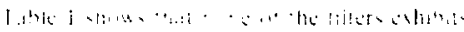

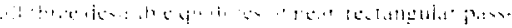

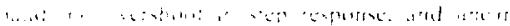

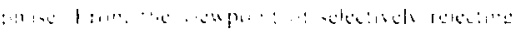

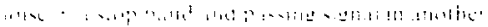

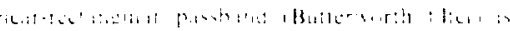

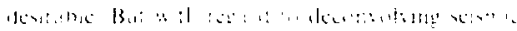

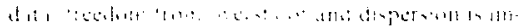

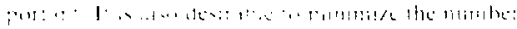

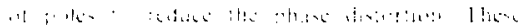

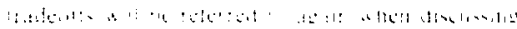

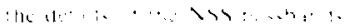

\section{(HAPTER 5: NSS BANDMPAST FIITFRS}

\section{RIVI) HITRS}

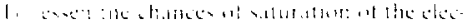

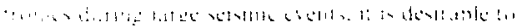

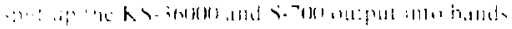

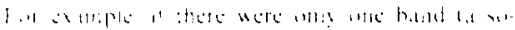

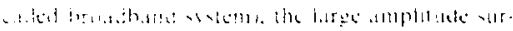

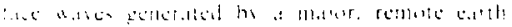

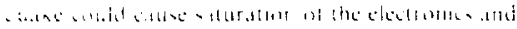

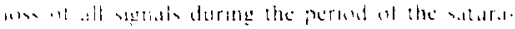

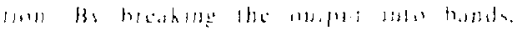

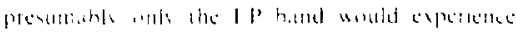

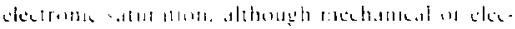

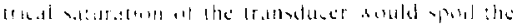

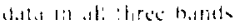

\section{SAVIMA FIIIFRS}

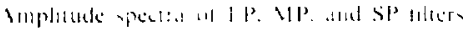

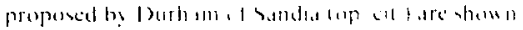

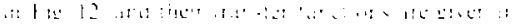
Bptas:

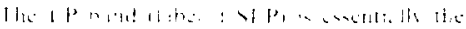

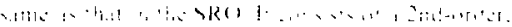

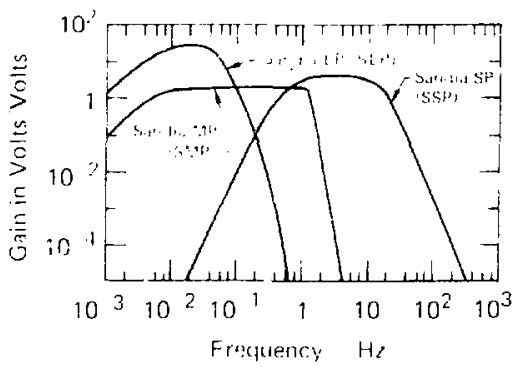

HC, 12. Sandia I.I', VII', and ST' laand filler amplitude upectri. 


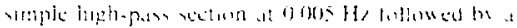

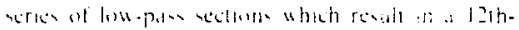

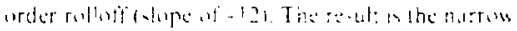

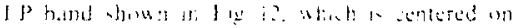
in: $11, i=1$ 51 $(4)$

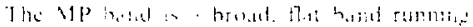

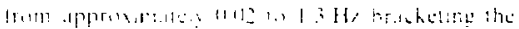

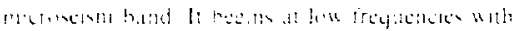

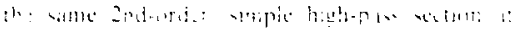

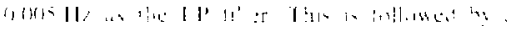

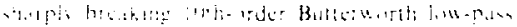

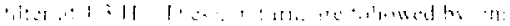

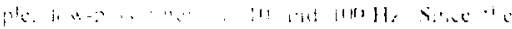

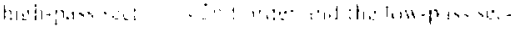

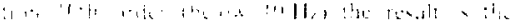

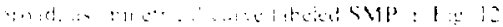

$$
1+1,0
$$

1111.1 .

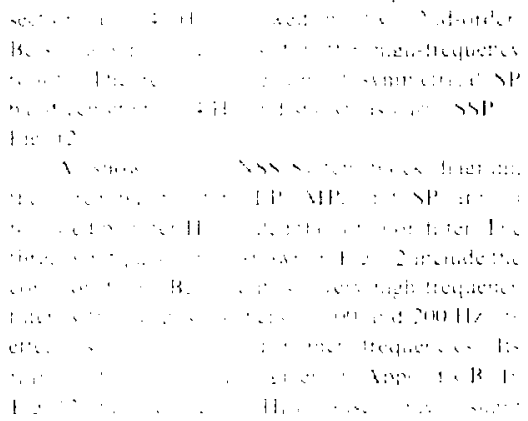

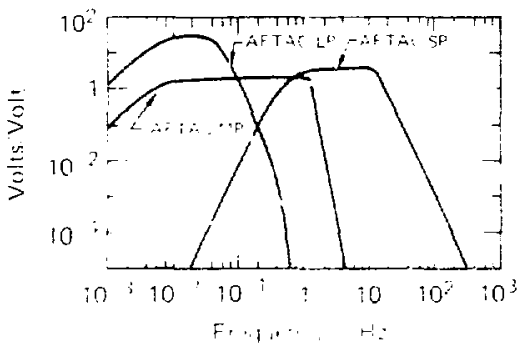

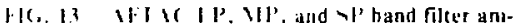
plitude spectra.

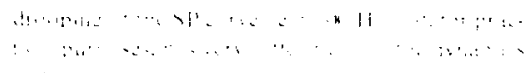

\section{IFIC MIITHS}

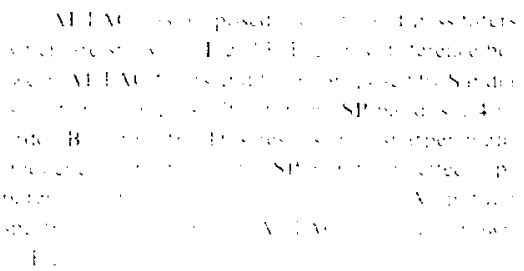

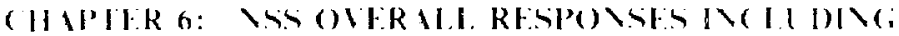

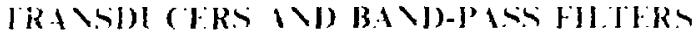

\footnotetext{
K. .

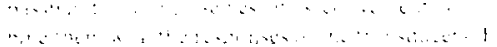

a i

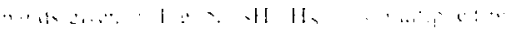

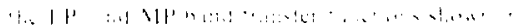

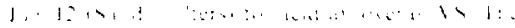

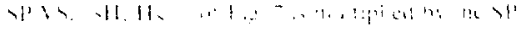

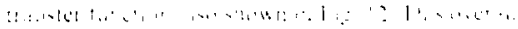

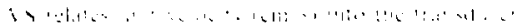

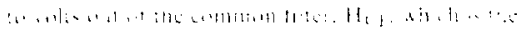

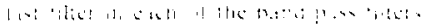

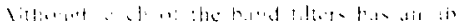

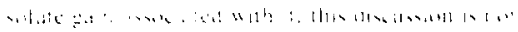

$+1$

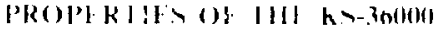

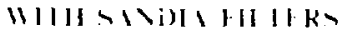

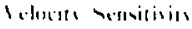

$1, \ldots+\cdots, \ldots, \cdots, \cdots$

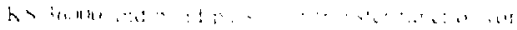
I

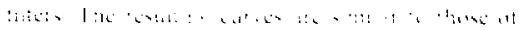




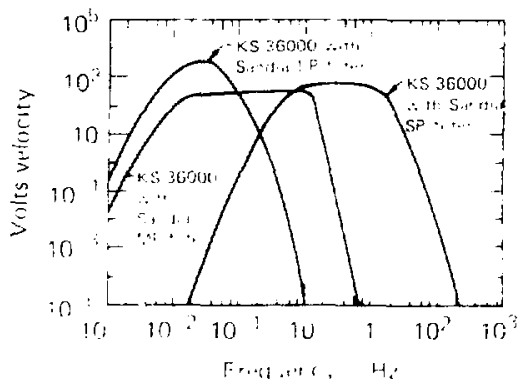

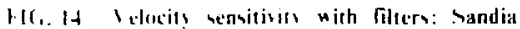

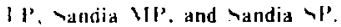

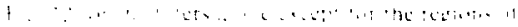

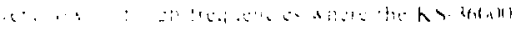

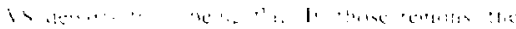

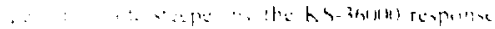

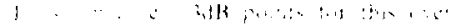

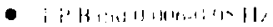

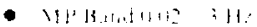

- y'is ind 1-1'11.

Phithe Kesponst if

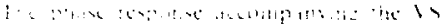

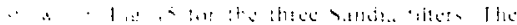

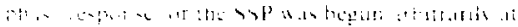

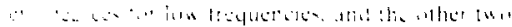

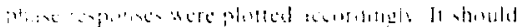

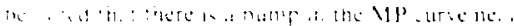

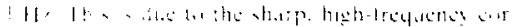

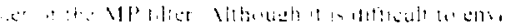

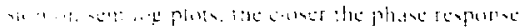

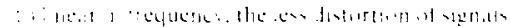
i.

\section{(, roup Ilelatis}

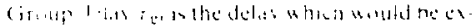

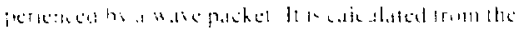

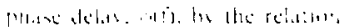

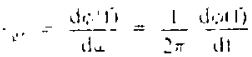

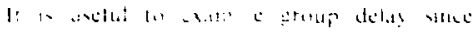

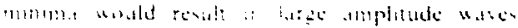

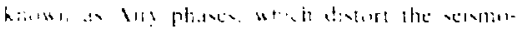

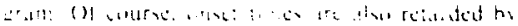

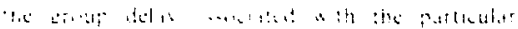

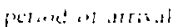

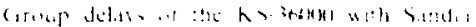

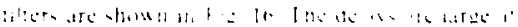

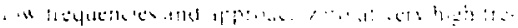

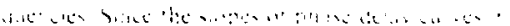
$\therefore$ a

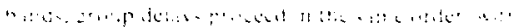

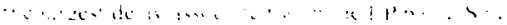

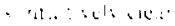

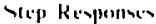
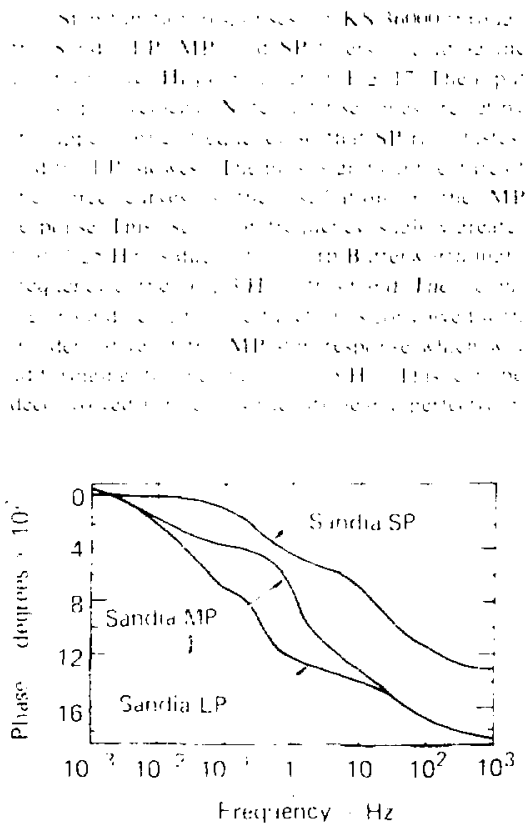

HIC; 15. Thase resporse of hi-361loll with sandia Filters. 


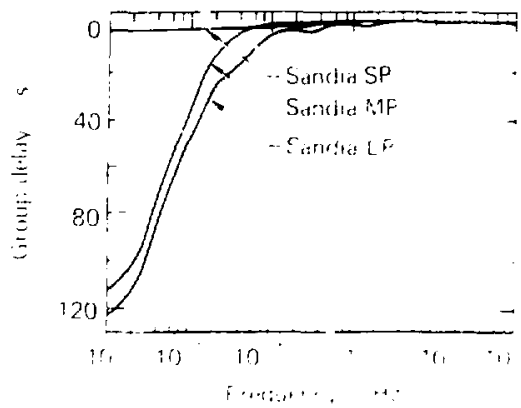

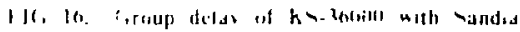
fillors.

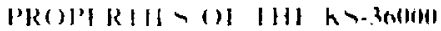

HIII H I H H H H

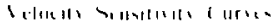

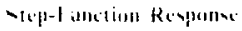

$1 ! \because, \because, \therefore+\cdots+\cdots, \therefore, \cdots$

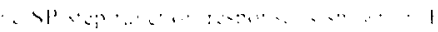

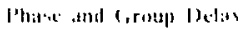

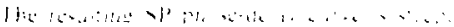

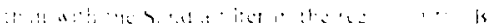

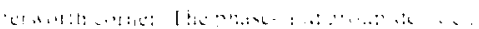

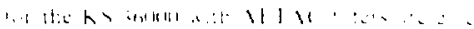

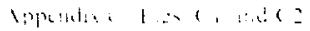

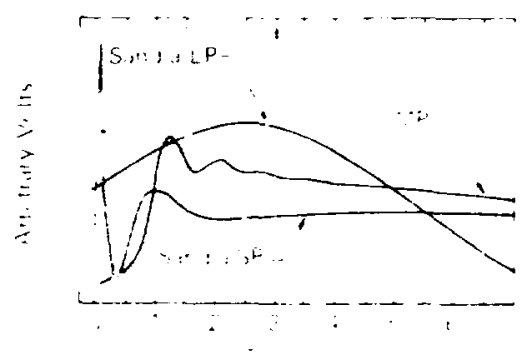

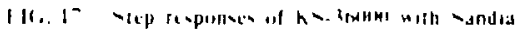
$-\cdot \therefore$

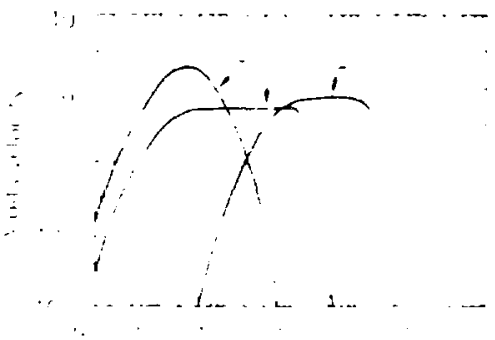

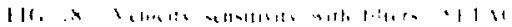

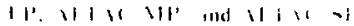

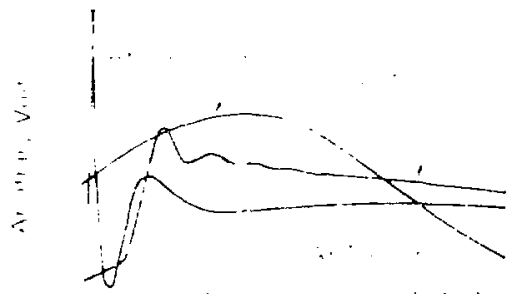

;

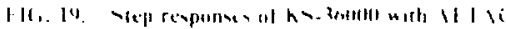
filter. 


\section{CHAPEER 7: THE MP-BAND FILTER}

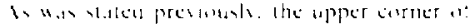

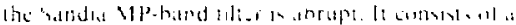

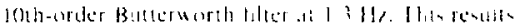

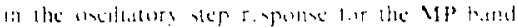

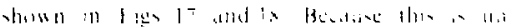

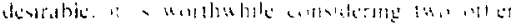

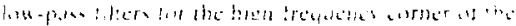

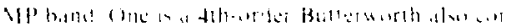

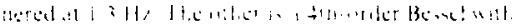

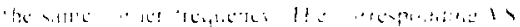

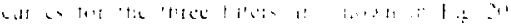

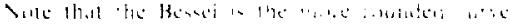

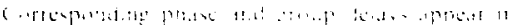

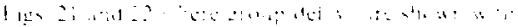

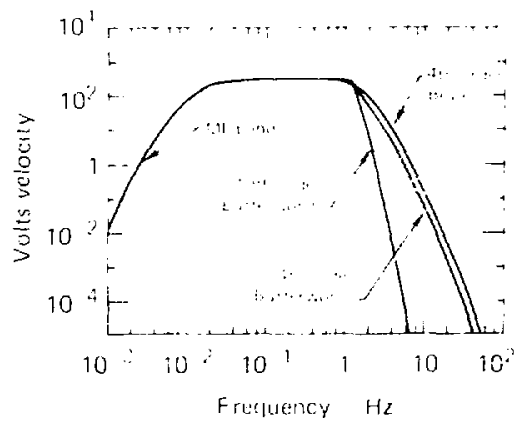

He. 211. Irequeres response comparison of three 11P hand lon pass filcers.

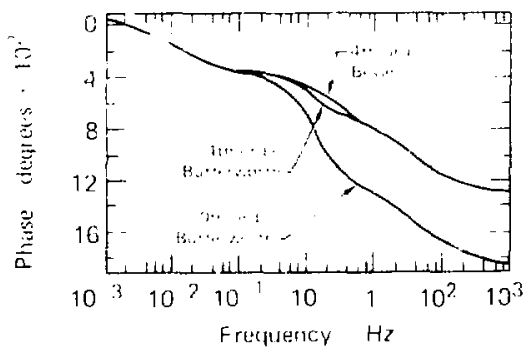

If i. 21, Jhase delay comparisun of thre. - :'band low pass filters.

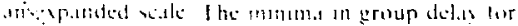

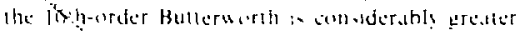

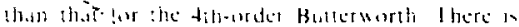

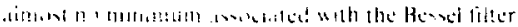

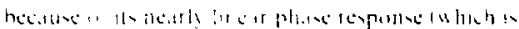

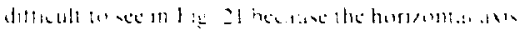

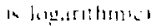

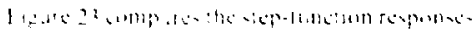

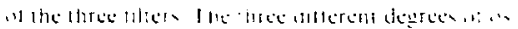

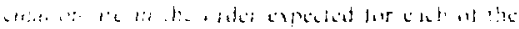

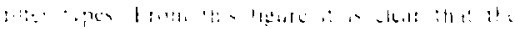

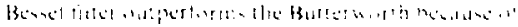

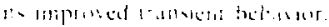

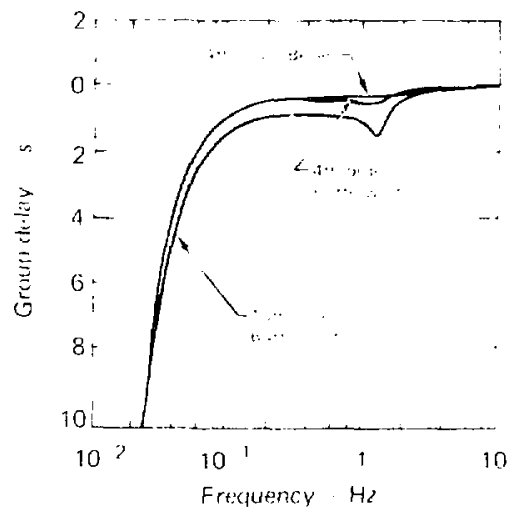

Hic.22. Corentp delat comparinon of three MIJ band low pass filters.

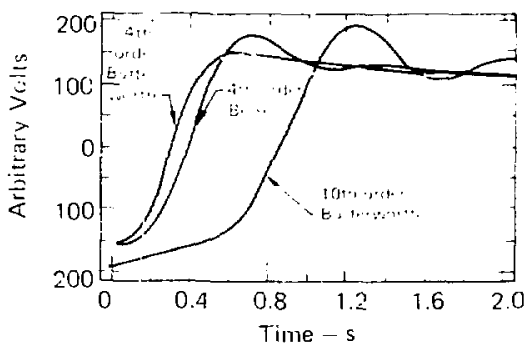

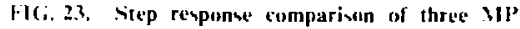
band kim pasa filters. 


\section{( HAPTER 8: THE KS-36000 RESPONSF \\ TO VAR!OIS REAL SEISIIC SOI RCES}

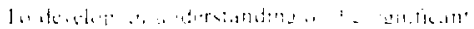

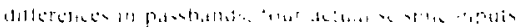

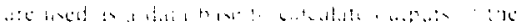

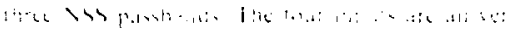

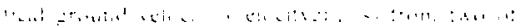

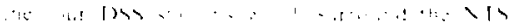

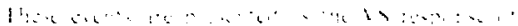

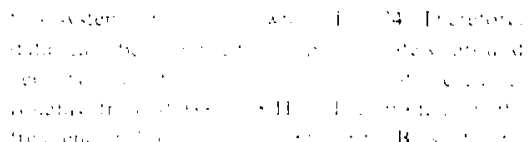
11

iv 1

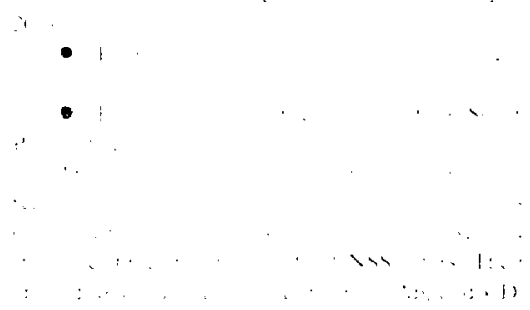

\section{$1 \cdots 11 ; \cdots \quad 1:: \ldots$}

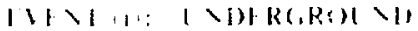

U( IIIR $\rightarrow H())+3=3.0^{\circ}$

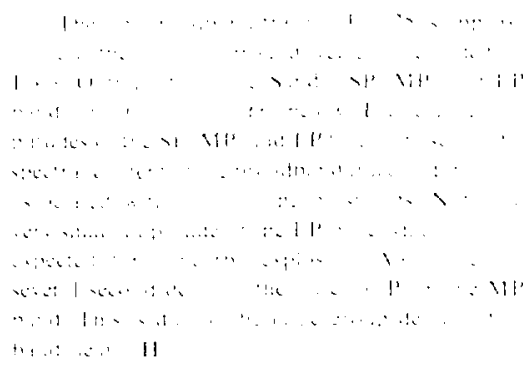

FIV I Ii): Rl(ill)

FIRIIIOL Iht I $=20^{\circ}$,

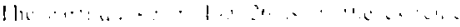

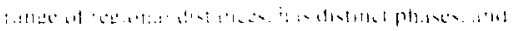

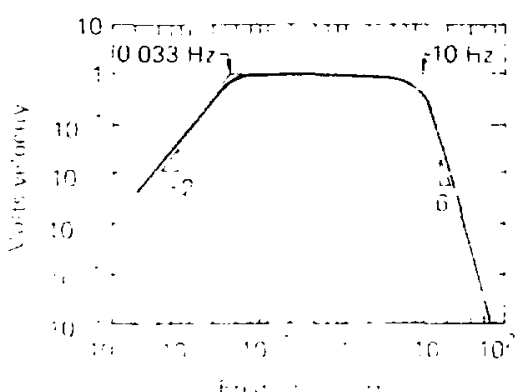

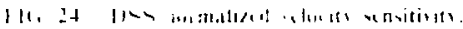

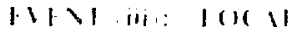

||$R|H()||h|$. I.

$11 !$

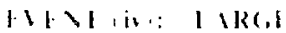

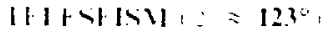

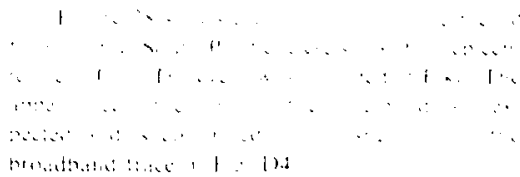




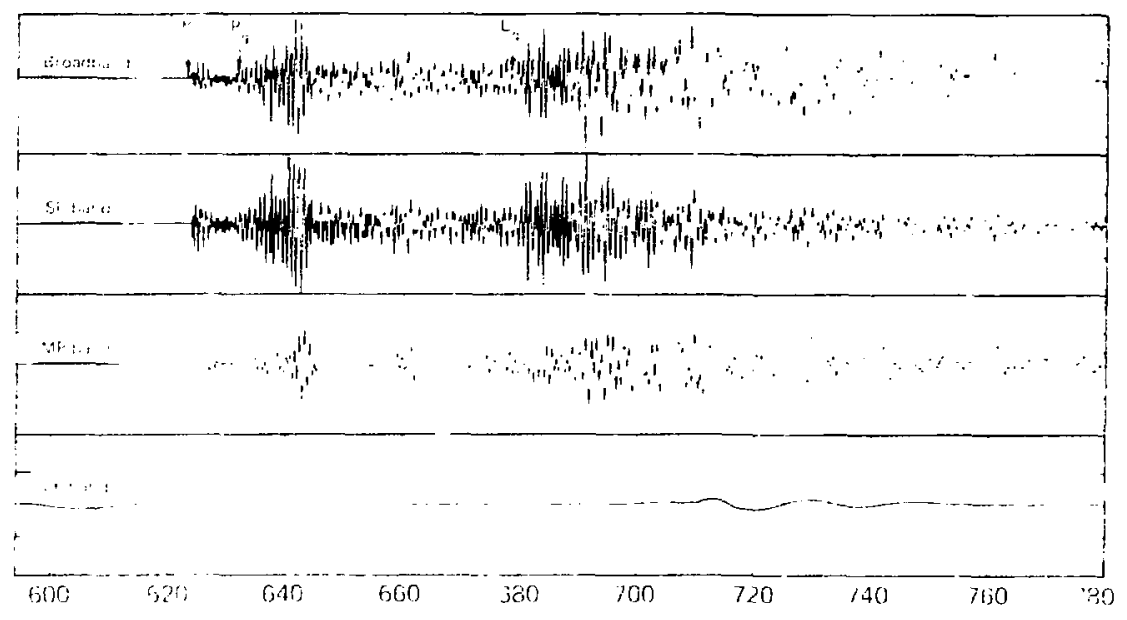

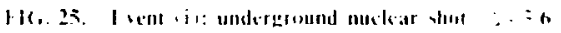

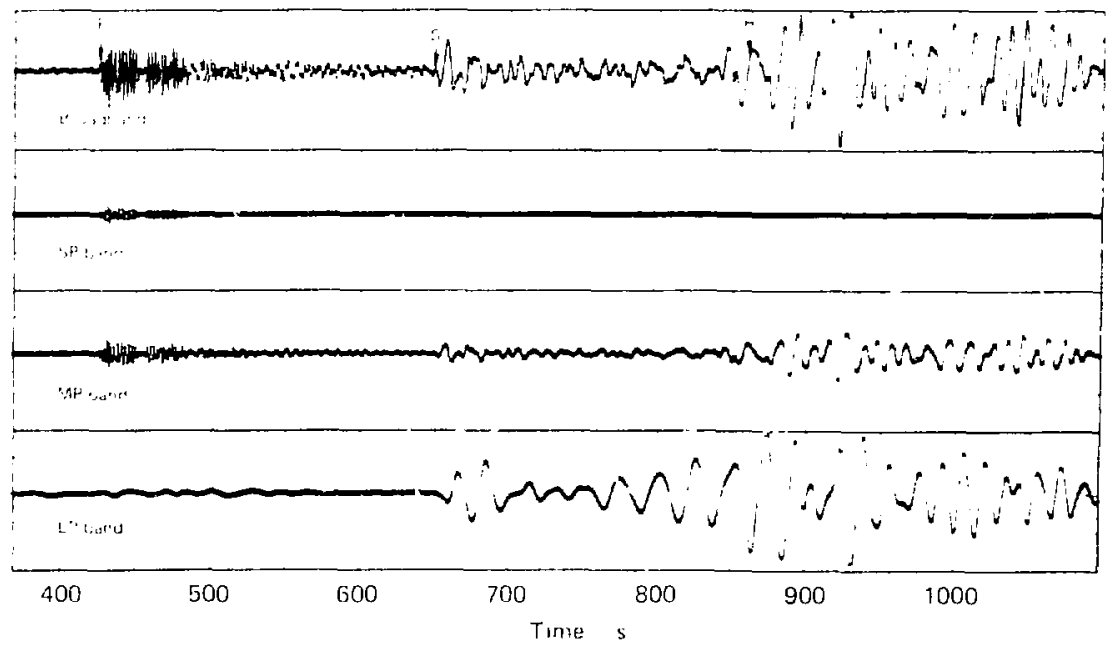

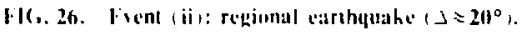




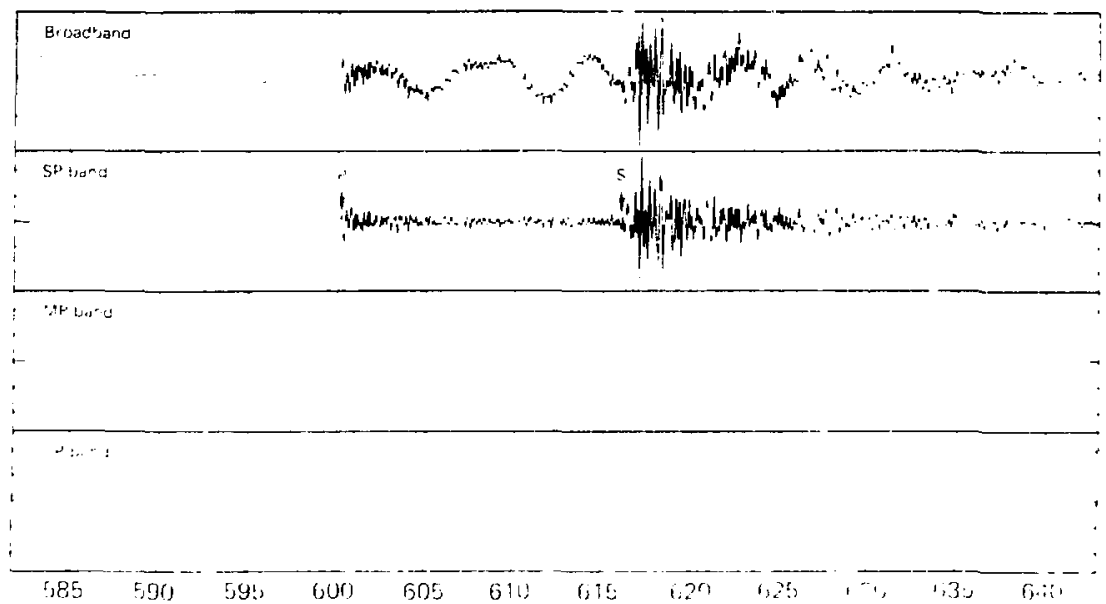

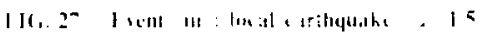

m 1

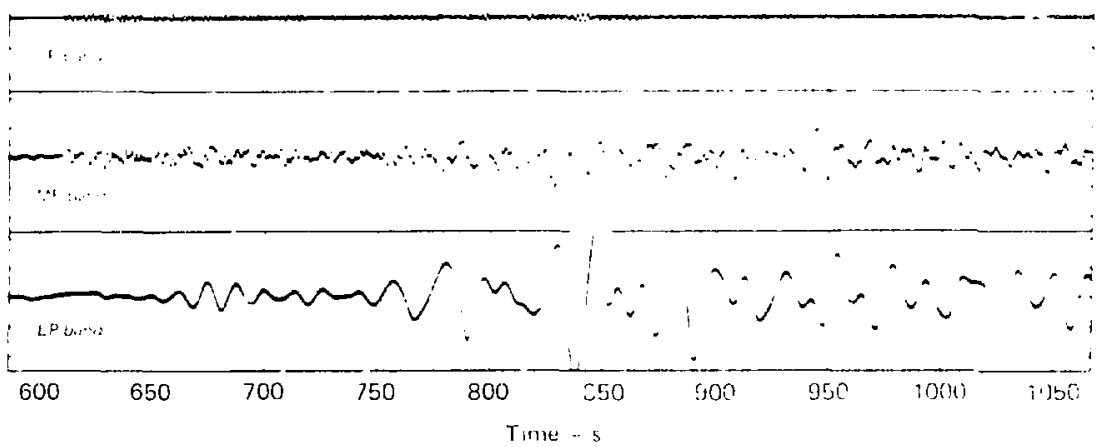

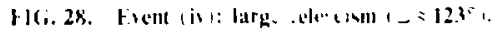



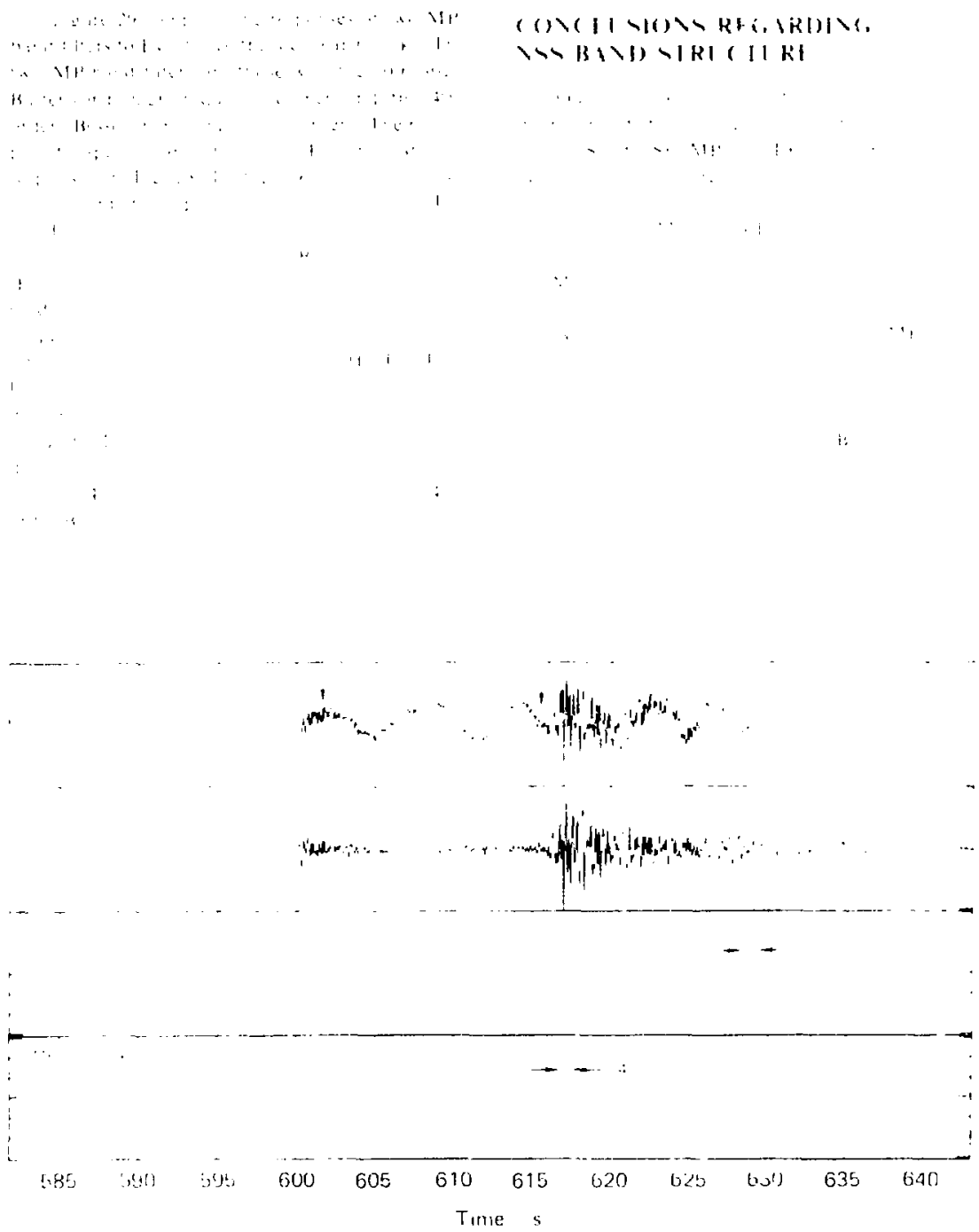

HIC. 29. Fffect of two MP filters on a local eurthquake. 


\section{CHAPIFR 9: THF S-7MO SISTFW}

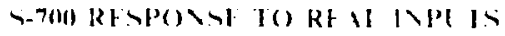

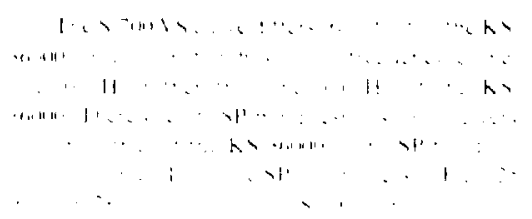

S-

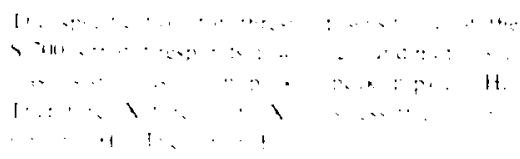

11. 


\section{ACKNOWLEDGMENT}

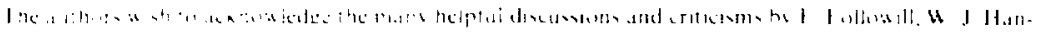

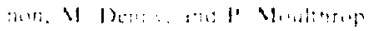




\section{APPFNDIX A: SFISMOMITHR TRANSFER FINCTIONS}

IRISSHFR FI Y IIOSS FOR THE RS-360MO SFISMOMHTHR

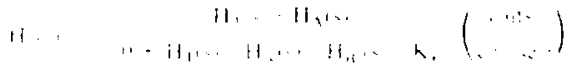

$$
\begin{aligned}
& 11 . \quad \ldots\left(\begin{array}{l}
\cdots \\
\therefore
\end{array}\right) \\
& \text { 11. } \cdot 11 \\
& \text { H.. } \quad . \quad\left[\begin{array}{ccc}
\cdot & & \\
\cdots & \ldots & .
\end{array}\right]\left(\begin{array}{l}
\cdots \\
\cdot
\end{array}\right) \\
& \text { h } \quad i \quad 1
\end{aligned}
$$

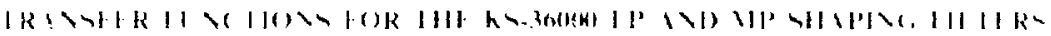

$$
\begin{aligned}
& \text { H. H... H. H }(\ldots)
\end{aligned}
$$

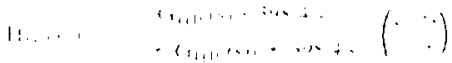

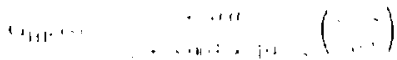

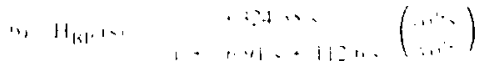




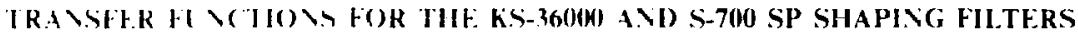

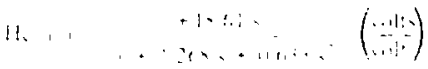

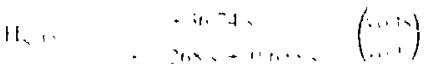

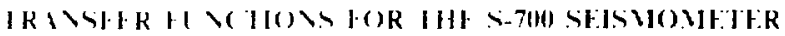

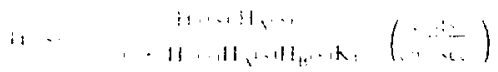

$11 \cdot \cdots\left(\begin{array}{lll} & \ddots \\ \ldots & \ldots\end{array}\right)$
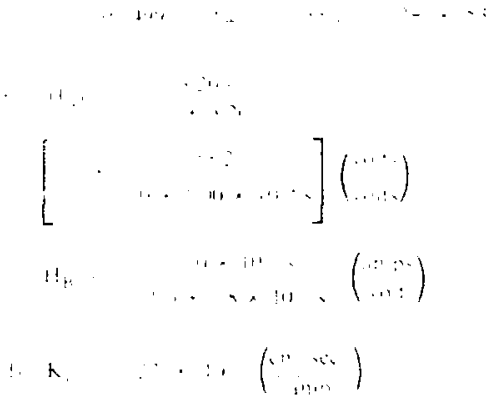

is 


\section{APPENDIX B: FITTER TRAYSFER FINCTIONS}

SHORT PFRIOI FIITHR TR I STER FI VCTIOSS

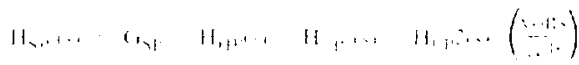

$\| \ldots \ldots[\ldots]$

in $H$. .

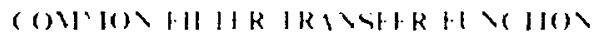

11. .

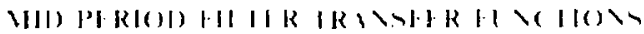

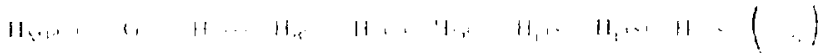

$11, \cdots$

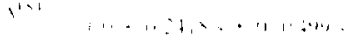

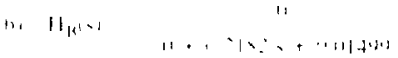




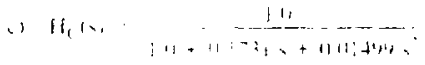

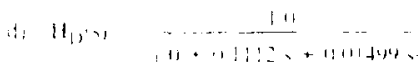

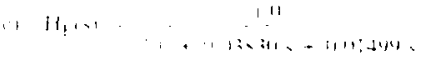

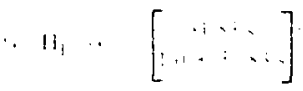

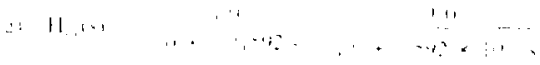

$119: 1$

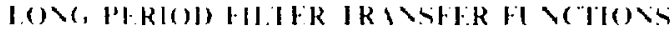

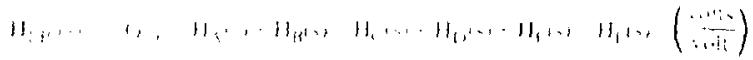

$$
\begin{aligned}
& 11,1 \ldots\left[\begin{array}{l}
\ldots \\
.1-\ldots 11, \cdots, 1 \cdots
\end{array}\right]
\end{aligned}
$$

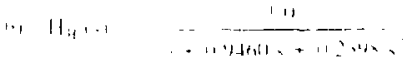

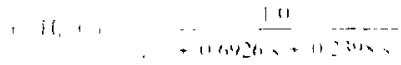

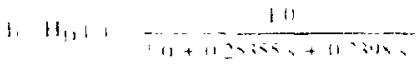

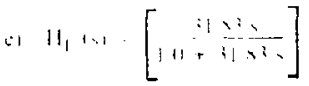

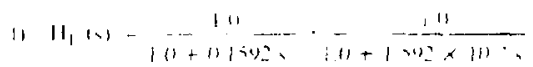

$$
\begin{aligned}
& \text { H) } C_{11}=3 . .11
\end{aligned}
$$




\section{APPENDIX (': PHASF AND GROIP DFIAI \\ WITH AFTAC FILTERS}

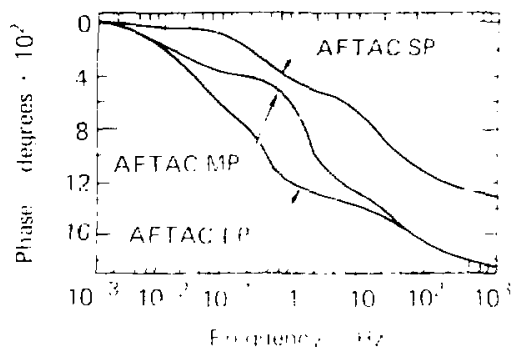

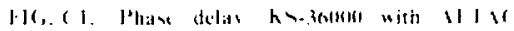
filters.

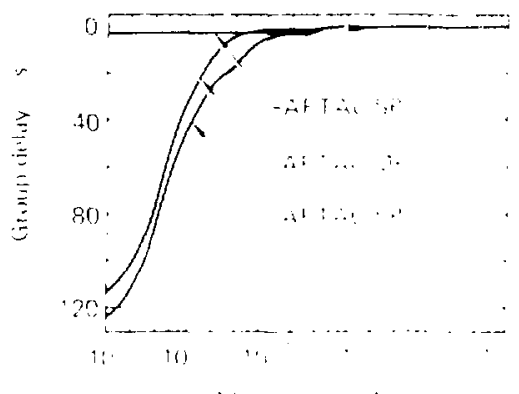

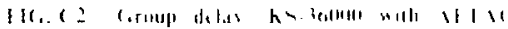
filters. 


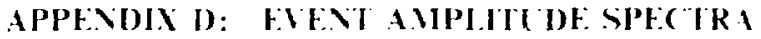

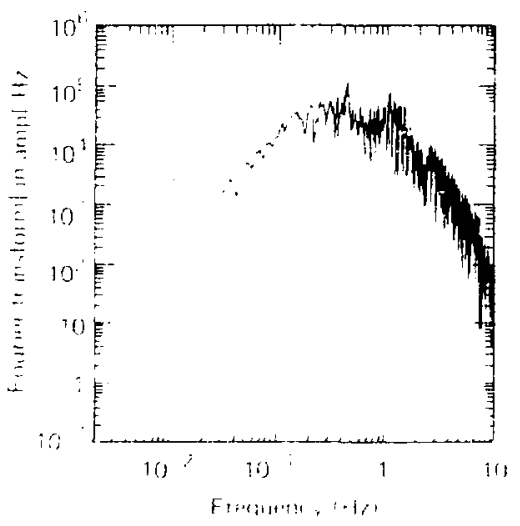

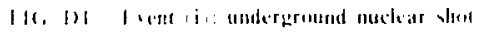
amplitida spectrum:

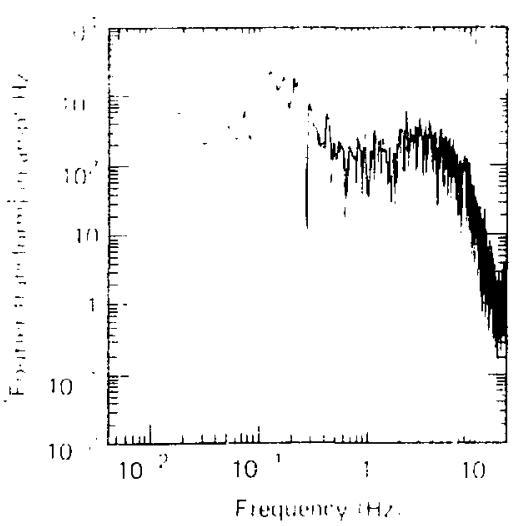

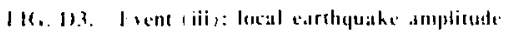
yectruni.

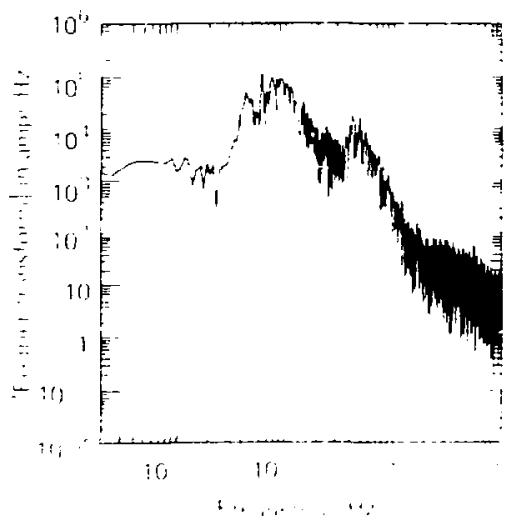

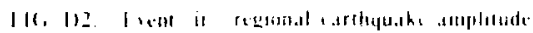
vientrum

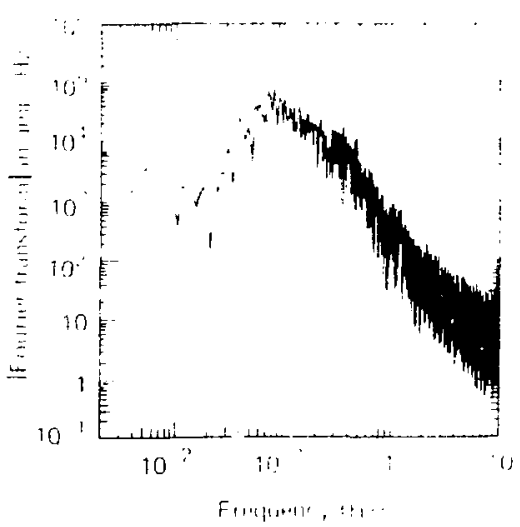

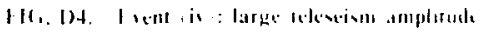
ypetistums. 